\title{
Learning From Demonstration and Interactive Control of Variable-Impedance to Cut Soft Tissues
}

\author{
Rui Wu ${ }^{\circledR}$ and Aude Billard ${ }^{\circledR}$, Fellow, IEEE
}

\begin{abstract}
In this article, we propose an approach to extract variable-impedance during cutting tasks from human demonstrations, so as to ease soft-tissue cutting by robots. We model the dynamic adjustment of the human arm during interactions with the tissue and transfer these adaptive capabilities to the robot, by learning both the motion and change of impedance. To improve performance during task execution, our variable-impedance skill-transfer framework combines the learned model with an interactive-operation and feedback controller. To offer the flexibility of modifying the trajectory at run time, we use a control law based on dynamical systems. We couple this control law with virtual dynamics that describes the cutting dynamics. This ensures that the robot can control the interaction force and can plan the trajectory. The approach is validated in a real robot cutting-experiment of cutting different hardness tissues. Results show that our learning-based feedback controller, with the assistance from interactive-operation models, can effectively improve the task's success rate, cutting length, cutting depth, and other indicators, as compared to using a fixed and variable-impedance gain controller.
\end{abstract}

Index Terms-Dynamical systems (DS), learning from demonstration (LfD), soft tissues, variable-impedance skill transfer.

\section{INTRODUCTION}

$\mathbf{H}$ UMANS have very powerful and flexible physical manipulation capabilities, especially when operating on objects

Manuscript received January 2, 2021; revised May 18, 2021 and July 27, 2021; accepted August 28, 2021. Recommended by Technical Editor C. Xiong and Senior Editor H. Qiao. The work of Aude Billard's was supported by the ERC Advanced under Grant SAHR. This work was supported in part by the EPFL-ZEISS Idea grant on "Al-Based Training of Robots for Surgical Task" and in part by the scholarship from the China Scholarship Council. Aude Billard's time was partly supported by the ERC under Advanced Grant SAHR. (Corresponding author: Rui Wu.)

Rui Wu is with the Learning Algorithms and Systems Laboratory, School of Engineering, École Polytechnique Fédérale de Lausanne, Lausanne, $\mathrm{CH}$ 1015, Switzerland, and also with the State Key Laboratory of Robotics and Systems, Harbin Institute of Technology, Harbin 150001, China (e-mail: rui.wu@epfl.ch).

Aude Billard is with the Learning Algorithms and Systems Laboratory, School of Engineering, École Polytechnique Fédérale de Lausanne, Lausanne, CH 1015, Switzerland (e-mail: aude.billard@epfl.ch).

This article has supplementary material provided by the authors and color versions of one or more figures available at https://doi.org/10.1109/TMECH.2021.3123356.

Digital Object Identifier 10.1109/TMECH.2021.3123356 that deform as a result of manipulation. To meet the needs of these manipulation tasks, humans can control well the subtle interaction process between their bodies and objects. Today, increasingly more researchers hope that robots will also be able to perform complex manipulation tasks with human-like dexterity. Although robotic manipulation has traditionally been restricted to rigid objects, such as pin-hole assembly [1], [2], recent works target the manipulation of deformable objects, such as when grating vegetables and scooping fruits [3], [4]. Large efforts are also conducted worldwide to enable surgical tasks to be performed, at least in part, by robots such as robot-assisted needle insertion [5], [6] and suturing [7]. Cutting tissues, be it that of a vegetable or human skin, is the equivalent of the peg-in-hole problem for rigid objects. As simple as it appears, this task is not trivial to implement and to control safely and accurately [8], [9].

State of the art of control of soft-tissues cutting: When cutting soft tissues, the object's deformation is substantially dependent both trajectory and interaction forces. Nonlinear interfacial friction and viscosity also make the cutting process more complicated [10]. The feedback method is widely used in dealing with the complex and unknown models, for example, visual-serving technology is used to update the cutting trajectory in real time [11] and to monitor the deformation process [12], [13] and, with a combined force/vision-control strategy [14] using both force and visual feedback, to adjust the cutting force and speed of a robot's operation. Learning-based methods also use haptic data to classify objects' physical properties and to change a robot's operation during the cutting [15]. In this article, we target single-layered skin-like soft tissue. During cutting, the tissue is fixed on a platform and stretched to reduce the cutting force and interfacial friction [8]. Our attention is focused on the cutting force generated by the contact between the blade and the tissue in the cutting direction and on the contact force perpendicular to the cutting direction. We also focus on learning human manipulation capabilities and collaboration abilities in order to help the robot meet the requirements of the changes, during actual operations, in complex objects' physical characteristics and, in complex interactive tasks, to improve the human-robot collaboration performance of robot. With these assumptions, we target our work to certain specific applications in industry such as for cutting meat, and robotic surgery-assistance.

Human approach to soft-tissue cutting and to control of impedance: In cutting operations, such as surgical cutting, 
skilled practitioners first ensure that the cut depth meets the requirements and that the length of the cut is sufficient. The cutting process also needs to be as stable as possible to reduce unexpected tissue damage [16]. After years of training, these skilled practitioners automatically control the movements of their hands, as demonstrated in professional welding [17]. The thought process involved in determining how to adjust the impedance and posture of the arm in order to cut at the desired depth, while ensuring stable force interaction, is quasi unconscious [18]. These skills are known in robotics as a variable impedance control [19], which was offered as a powerful approach to mitigating the uncertainties of contact [20].

Requirements for robot control of soft-tissue cutting: To enable a robot to autonomously and effectively perform a complex interactive operation, two different issues need to be considered: First, the robot needs to plan the appropriate/desired trajectory for the task. Second, it must embed a model of how the impedance changes along the trajectory and as a result of the interaction forces [21]. For the learning of robot trajectories, one approach we follow is to learn the trajectory demonstrated by humans in order to generate an impedance-based dynamical systems controller [22], [23]. This ensures the generation of a motion model that can recover, on the fly, from external perturbations and can return to the desired trajectory. The shaping of the impedance enables the robot to determine from which direction the controller will accept perturbations and from which direction it will reject them. The impedance-parameter changes can be learned via human demonstrations [22], [24] or estimated by the human arm electromyography (EMG) signals [25], [26] or through reinforcement learning [27].

Our contributions: In this article, we extend these previous approaches and build a human-skill transfer framework that can transfer both human-robot collaboration capabilities and dynamic adjustment capabilities to interactive operations under a variable impedance control mode. First, robots need to have the ability to quickly imitate human operations and to adapt to human-robot interactions and collaborations. To address this need, we propose a coupled dynamical system (coupled DS) model for interactive tasks: It implements different motion modes under a unified DS model in order to adapt, during robot's operation, to the interruptions and interferences of the user. To ensure the integrity of the cut, the DS model we propose can accurately control the robot to return to where it was when the user stopped it (see Fig. 1). Second, as a tissue to be cut can never be completely known, we posit that it is important to learn the bounds on what reasonable impedance and forces should be adopted. Most importantly, in response to change in the interaction forces, we must embed a model of modification of the velocity and impedance. To compensate and modify the trajectory, when faced with different tissues' responses, the hidden causal relationship of "position-force," "force-impedance," and "force-velocity" in human interactive operations are learned as interactive-operation models (IO models) and used in a velocity-adjustment (VA) and DS-modulation strategies. This enables us to compensate for position error and to restrain the force penetration. Third, to adapt to changes in interaction forces, by using the abovementioned learning model, we propose


Fig. 1. Compliant robot automatically performing a cutting task. A human pulls the robot away when the robot approaches the tissue, because they want to pause the action of the robot (top left); then, during the cutting, they pull the knife away from the tissue to check the status of the cutting trajectory (top right and bottom left). The robot can perform a safe and stable interaction with a human and, after the human leaves, the robot will return to the point of disturbance and continue its work.

a force feedback-based variable-impedance controller (FFBVI controller).

As the framework that we propose is designed to solve the force-interaction problem with unknown environments, the passivity or stability of the system must be guaranteed [28], [29]. A controller that ensures a passive relation, between external forces and a robot's velocity, will yield stable behavior in free motion and in contact with any passive environment [30]. To ensure the passivity of the entire system, our method incorporates a special controller based on a selective dissipation of energy in directions that are irrelevant to the task [31].

We evaluate our framework in a robot cutting-task, under different task conditions such as a change in tissue's hardness. The results show that our proposed framework can automatically adapt to a certain degree of task-condition changes and can ensure the improvement in performance indicators, such as cutting length, cutting depth, maximum contact-force peak, and task success-rate.

\section{METHOD}

\section{A. Rigid Body Dynamics and the Passive DS Controller}

We start from the rigid-body dynamics of an $N$ degrees-offreedom manipulator in Cartesian space

$$
M\left(x_{r}\right) \ddot{x}_{r}+C\left(\dot{x}_{r}, x_{r}\right) \dot{x}_{r}=F_{c}+F_{e}
$$

where $\boldsymbol{M}\left(\boldsymbol{x}_{\boldsymbol{r}}\right), \boldsymbol{C}\left(\dot{\boldsymbol{x}}_{\boldsymbol{r}}, \boldsymbol{x}_{\boldsymbol{r}}\right) \in \mathbb{R}^{3 \times 3}$ are the inertia matrix and Coriolis/centrifugal matrix, the $\boldsymbol{x}_{\boldsymbol{r}}, \boldsymbol{F}_{\boldsymbol{c}}, \boldsymbol{F}_{\boldsymbol{e}} \in \mathbb{R}^{3}$ are, respectively, the real Cartesian position of the robot end-point, the control force, and the external force. Assume that we have at our disposal a low-level controller for inverse kinematic and gravity compensation, ${ }^{1}$ so the gravity forces $\boldsymbol{g}(\boldsymbol{x}) \in \mathbb{R}^{3}$ are already compensated.

The passive DS controller [31] will generate torque for driving the robot to follow the desired velocity:

$$
\begin{aligned}
\boldsymbol{F}_{c} & =D\left(x_{r}\right)\left(\dot{x}_{d}-\dot{x}_{r}\right) \\
\boldsymbol{D}\left(x_{r}\right) & =Q\left(x_{r}\right) \Lambda Q\left(x_{r}\right)^{T} .
\end{aligned}
$$

\footnotetext{
${ }^{1}$ As this is the case for the KUKA robot used in the implementation.
} 




Fig. 2. Human demonstration is divided into three phases, based on the movement characteristics. The changing laws of velocity, force, and impedance are obviously different, thus revealing some characteristics of the whole operation process.

As the desired motion in our DS is given by $\dot{\boldsymbol{x}}_{\boldsymbol{d}}=f\left(\boldsymbol{x}_{r}\right)+$ $f_{n}\left(x_{r}\right)$, the $f\left(x_{r}\right)$ is desired DS motion and the $f_{n}\left(x_{r}\right)$ is a learning-based DS-modulation, the $e_{1}, \ldots e_{N}$ is an orthonormal basis with the $e_{1}=\frac{f\left(x_{r}\right)}{\left\|f\left(x_{r}\right)\right\|}$ along the direction of desired motion and $e_{2}$ is rotated $90^{\circ}$ around the normal of the cutting-motion plane relative to $e_{1} . e_{1}, \ldots e_{N}$ are the columns of the matrix $Q\left(x_{r}\right)$ and $\Lambda$ is a diagonal matrix with eigenvalues $\lambda_{1}, \ldots \lambda_{N}$ that represent the damping of a robot controller.

With the passive DS controller, both the desired velocity and damping can be specified to make the robot show the desired performance. Normally, when we learn a skill from human demonstrations, we focus on the model of the velocity-position relation of the demonstration trajectory. This kind of model, similar to stable estimator of dynamical systems (SEDS) and locally active — globally stable dynamical systems (LAGS-DS), is proposed in [32] and [33]. These DS models can ensure the model is globally asymptotically stable and always converges to the target point; and to reproduce the human motion, it cannot only properly generate velocity, based on the state of the robot, but also replan the trajectory very rapidly to adapt the changes of task conditions [34], [35]. Whereas, for the force-interaction tasks, to make sure the robot can appropriately interact with the environment, not only the velocity needs to be adjusted based on the robot state, but the impedance performance should also be controlled. Therefore, in the following sections, two kinds of models are learned from human demonstration and for robot motion-control and interaction control, we named them "motion model" and "IO model."

\section{B. Learning the DS Motion Model From Human}

To more accurately extract features of human cutting-skills, we divide the entire cutting task into the following three phases (see Fig. 2).

1) Phase 1 is the approach-penetration phase. In this phase, the knife will approach the surface of the tissue and the knife will pierce into the tissue.

2) Phase 2 is the cutting phase: The knife will cut the tissue and ensure the cutting depth and length.

3) Phase 3 is the leaving phase. In this phase, the robot will finish the cut and pull the knife out of the tissue.

During phase 1, the robot should overcome the uncertainty linked to the position of the contact surface and should control the interaction force of the penetration. During phase 2, to cut through the tissue, the robot should maintain a vertical contact
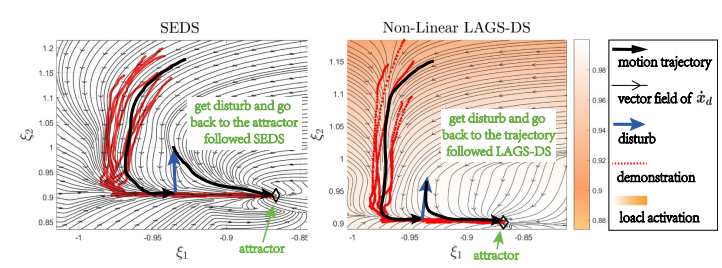

(a)



(b)

Fig. 3. Compare among SEDS (up left) LAGS-DS (up right) and coupled DS (below). The coupled DS can return to the disturbance point and can continue the cutting task, i.e., not only follow a fixed-flow field. It more realistically imitates the human operation process and can reflect the operating characteristics of the operator.

force with the tissue; then to finish the cutting action, it must overcome the interaction force along the cutting direction.

In this article, we propose a unified DS model for realizing the entire motion process that can ensure the generalization performance of the model and the performance against disturbances.

1) Novel Coupled DS Model: Our model is different from other DS motion models (as shown in Fig. 3): It is composed of three coupled DS models that (denoted DS1, DS2, and DS3 for each of the three phases) are learned from demonstration data, after phase separation. DS1 is used for phase 2 to execute the cutting motion; and DS2 can be used for phase 1 to approach and penetrate the tissue or for phase 2 to return to the original point of disturbance. DS2 has a mobile attractor that is based on a virtual DS and that moves along the desired cutting trajectory. DS3 has only one function: to remove the knife from the tissue.

For DS1, a DS model is built along the cutting direction, and the DS1 will be activated when DS2 arrives at its target; the position data of the robot will be transferred into the state value $\boldsymbol{x}_{r}$; and the output of DS1 $\dot{\boldsymbol{x}}_{1} \in \mathbb{R}^{3}$ will give the robot velocity command

$$
\left\{\begin{array}{l}
\dot{x}_{1}=f_{\mathrm{DS} 1}\left(\boldsymbol{x}_{\boldsymbol{r}}\right)=\left(\boldsymbol{A}_{\mathbf{1}} \cdot \boldsymbol{x}_{\boldsymbol{r}}+\boldsymbol{b} \mathbf{1}\right) \cdot \delta\left(\boldsymbol{\xi}, \boldsymbol{x}_{\boldsymbol{r}}\right) \\
\boldsymbol{x}_{\boldsymbol{r}}{ }^{*}=\boldsymbol{A}_{\mathbf{1}}^{-1} \cdot \boldsymbol{b}_{\mathbf{1}}
\end{array}\right.
$$

where $A_{1} \in \mathbb{R}^{3 \times 3}$ are the negative-gain diagonal matrices, $\boldsymbol{b}_{\mathbf{1}} \in$ $\mathbb{R}^{3}$ can be calculated after reaching the target point $\boldsymbol{x}_{\boldsymbol{r}}{ }^{*}$.

The DS2 will run when the robot is in the phase 1 or when a disturbance is detected in phase 2 . Hence, its model will be combined with the DS1, and the attractor of DS2 is forced to keep moving with the state of DS1. This ensures that the knife will return to the point of separation caused by the disturbance. 
When DS2 is activated, the robot position will transfer into the state value $\boldsymbol{\xi} \in \mathbb{R}^{3}$ and the output will be the robot velocity command

$$
\left\{\begin{array}{l}
\dot{\xi}_{d}=f_{\mathrm{DS} 2}(\xi)=A_{2} \cdot \xi+b_{2}\left(\xi^{*}\right) \\
\xi^{*}=A_{2}^{-1} \cdot b_{2}
\end{array}\right.
$$

The switch condition based on disturbance is

$$
\delta\left(\boldsymbol{\xi}, \boldsymbol{x}_{\boldsymbol{r}}\right)=\left\{\begin{array}{lll}
0 & \text { and } \boldsymbol{\xi}^{*}=\boldsymbol{\xi}_{m}^{*} & \text { if }\left\|\left(\boldsymbol{\xi}-\boldsymbol{\xi}_{m}^{*}\right)^{T} \cdot e_{2}\right\| \geq \varphi \\
1 & \text { and } \boldsymbol{\xi}_{m}^{*}=\boldsymbol{x}_{\boldsymbol{r}} & \text { if }\left\|\left(\boldsymbol{\xi}-\boldsymbol{\xi}_{m}^{*}\right)^{T} \cdot e_{2}\right\|<\varphi
\end{array}\right.
$$

in the real-time calculation of this formula, for each loop, the robot position $\boldsymbol{x}_{\boldsymbol{r}}$ at this loop of the DS1 is stored in $\boldsymbol{\xi}$, and the robot position at the last loop is recorded and transferred to the moving target-point $\boldsymbol{\xi}_{m}^{*}$ in DS2. To find out if there exist any human disturbances beyond the threshold $\varphi,\left\|\left(\boldsymbol{\xi}-\boldsymbol{\xi}_{m}^{*}\right)^{T} \cdot e_{2}\right\|$ calculates the distance between these two positions, in $e_{2}$ direction. When DS2 is activated, the moving target-point $\xi_{m}^{*}$ will be used as the target-point $\xi^{*}$ in DS2 and $\xi_{m}^{*}$ will not be refreshed. The $\boldsymbol{A}_{\mathbf{2}} \in \mathbb{R}^{3 \times 3}$ are the negative-gain diagonal matrices that drive the $\boldsymbol{\xi}$ back to the target-point $\xi^{*}$ that is also the point of disturbance $\boldsymbol{\xi}_{m}^{*}$; the $\boldsymbol{b}_{\mathbf{2}} \in \mathbb{R}^{3}$ are calculated by the target point $\boldsymbol{\xi}^{*}$.

For phase 3, the motion direction is to point away from the tissue, hence, DS3 can remove the knife from the tissue when the cutting is finished. And the DS3 $\dot{\xi}_{d \mathbf{1}}=f_{\mathrm{DS} 3}\left(\xi_{1}\right)=A_{\mathbf{3}} \cdot \xi_{1}+$ $\boldsymbol{b}_{\mathbf{3}}\left(\xi_{1}^{*}\right)$ (similar with DS2) will be triggered only when DS1 has arrived at its target point. Due to the coupling nature of the DS model proposed here, $f_{\mathrm{DS} 1}, f_{\mathrm{DS} 2}$, and $f_{\mathrm{DS} 3}$ are collectively referred to as $f_{\text {coupleDs }}$.

Note that the coupled DS has three DSs and three state values: $\boldsymbol{x}_{\boldsymbol{r}}, \boldsymbol{\xi}, \boldsymbol{\xi}_{\mathbf{1}} \in \mathbb{R}^{3}$. The real robot position-state will transfer into different state values and run different DSs, according to the disturbance and actual situation.

2) Learning the DS Models: To model and generate nonlinear motion, as required for the task and demonstrated by the human, DS1, DS2, and DS3 are trained as SEDS models; the $\boldsymbol{A}_{\mathbf{1}}, \boldsymbol{A}_{\mathbf{2}}, \boldsymbol{A}_{\mathbf{3}} \in \mathbb{R}^{3 \times 3}$ and $\boldsymbol{b}_{\mathbf{1}}, \boldsymbol{b}_{\mathbf{2}}, \boldsymbol{b}_{\mathbf{3}} \in \mathbb{R}^{3}$ are calculated by an SEDS algorithm and target point $\boldsymbol{x}_{r}{ }^{*}, \boldsymbol{\xi}^{*}, \boldsymbol{\xi}_{1}{ }^{*}$. For the motion of approach in phase 1 , the $\boldsymbol{\xi}^{*}$ is the predefined cutting starting point; and for returning the knife to the point of disturbance, the $\boldsymbol{\xi}^{*}$ is the disturbance point that is given by $\boldsymbol{\xi}_{m}^{*}$.

The combined three-layer coupled DS model proposed earlier builds upon and extends previous DS models proposed by our laboratory (SEDS [32] and LAGS-DS [33]). The differences are compared through numerical simulation in MATLAB and illustrated in Fig. 3. As we can see in Fig. 3, we reuse SEDS' guarantees that the trajectory converges to the last point on the cutting trajectory (attractor). But, whereas SEDS does not guarantee the return to the cutting trajectory, our model does [compare Fig. 3(a) left and (b)]. This might seem similar to the LAGS-DS approach that guarantees that the system will return to the closest point on the cutting trajectory. However, in LAGS-DS, this closest point could be further away from the point where the robot was stopped, hence, LAGS-DS cannot ensure that the tissue is properly cut along its entire length, whereas the coupled DS model guarantee that the system returns to the last cutting point [compare Fig. 3(a) right and (b)].



(a)

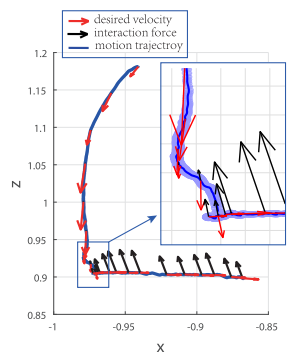

(b)
Fig. 4. (a) Force-impedance model can change the impedance of the robot when interaction force changes. When the interaction force increases (horizontally cut part), the higher impedance (bigger greed ellipse) can help the knife overcome resistance and (b) force-velocity model can control the velocity (red arrow) of SEDS, based on the interaction status during phase 1 , for instance, when the robot feels a contact force (black arrow), the velocity will decrease.

\section{Learning IO Model and VA Strategy}

We now explain how to extract and to use the information on force and impedance, in the demonstration for learning the IO models. The models will be used into two interaction-operation strategies and a feedback-control law based on human cognition for the robot execution.

1) Position-Force Model and Force-Impedance Model: After analysis of the demonstration data (as shown in Section IIIA), we can see that the interaction force is active only when the knife has a physical interaction with the tissue. Therefore, the mapping between position and force is learned and used for generating the desired force, based on the robot's position; a Gaussian mixture regression (GMR) [36] is used in this model. To make sure the knife continues to cut, a human would adjust their arm impedance, based on interaction force during cutting. To learn this ability from humans, we train a force-impedance model for robot variable-impedance adjustment; due to the nonlinear and unevenly distributed data of force-impedance relation, a Gaussian process regression (GPR) [37] model is used.

In the position-force GMR model, the input is the robot's position $\boldsymbol{x}_{\boldsymbol{r}} \in \mathbb{R}^{3}$, and output is the desired force $\boldsymbol{F}_{\boldsymbol{d}} \in \mathbb{R}^{3}$. The input and output of the force-damping GPR model is the interaction force $\boldsymbol{F}_{\boldsymbol{r}}$ and the desired impedance gains $\boldsymbol{d}_{\boldsymbol{d}} \in \mathbb{R}^{3}$, respectively. The $\boldsymbol{d}_{\boldsymbol{d}}=\left[d_{x} ; d_{y} ; d_{z}\right]$ represents the length of the three semiaxes $(X, Y, Z$ direction in Cartesian space) of the stiffness ellipsoid in human-arm impedance that is estimated by human-arm posture and EMG signals [38]. Fig. 4(a) shows the schematic of the force-impedance model.

Therefore, the models' output will be

$$
\begin{aligned}
\boldsymbol{F}_{\boldsymbol{d}} & =\boldsymbol{f}_{\mathrm{gmr} \boldsymbol{F}}\left(\boldsymbol{x}_{\boldsymbol{r}}\right) \\
\boldsymbol{D}_{\boldsymbol{d}} & =\operatorname{diag}\left(\boldsymbol{f}_{\mathrm{gpr} \boldsymbol{D}}\left(\boldsymbol{F}_{\boldsymbol{r}}\right)\right)
\end{aligned}
$$

where we obtain $\boldsymbol{F}_{\boldsymbol{d}} \in \mathbb{R}^{3 \times 1}$ and $\boldsymbol{D}_{\boldsymbol{d}} \in \mathbb{R}^{3 \times 3}$, and the $\boldsymbol{D}_{\boldsymbol{d}}$ is a diagonal matrix, and its diagonal elements are the three damping parameters generated by the GPR model.

2) Force-Velocity Model and Learning-Based VA Strategy: The target of the approaching motion is the initial contact position for the cutting task. In our case, we consider that the 


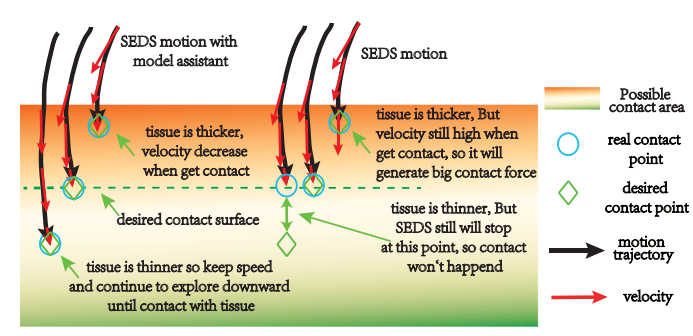

Fig. 5. VA strategy for the contact detection can change the DS model and velocity in phase 1 , based on different contact situations.

actual contact position in the vertical direction will change due to the inaccuracy of the robot motion and to the changes in the tissue's thickness.

When humans face a target change caused by a different thickness of tissue or inaccurate position information, they will change the stopping point of the approach phase, based on their judgement that comes mostly from force feedback. Whereas, for the robot running a DS model, it will follow only the target point we set. It necessary to use feedback force to detect the contact situation, as humans do and, based on the situation, to change the DS2 motion generator. Therefore, from human demonstrations, we learn the force-velocity model and propose a learning-based VA strategy in phase 1 to make sure the knife will approach and penetrate a tissue and to correct the error of the tissue's position or thickness.

Using the demonstration data in phase 1, we can learn the force-velocity model, based on the force-velocity relation in demonstrations with GMM-GMR algorithm, as follows. The model is shown in Fig. 4(b)

$$
v_{d}=\boldsymbol{f}_{\mathrm{gmr} \boldsymbol{V}}\left(\boldsymbol{F}_{\boldsymbol{r}}\right)
$$

where the $v_{d}$ is the magnitude of velocity.

Based on the force-velocity model $\boldsymbol{f}_{\mathrm{gmr} \boldsymbol{V}}\left(\boldsymbol{F}_{\boldsymbol{r}}\right)$ that we learned, the VA strategy is designed for changing the velocity magnitude of the DS2 model and provides a compensation velocity when the robot does not detect the contact in the target point $\boldsymbol{x}_{\boldsymbol{r}}{ }^{*}$

$$
\begin{aligned}
\boldsymbol{f}_{\boldsymbol{F} 2 \boldsymbol{V}}\left(\boldsymbol{x}_{\boldsymbol{r}}\right) & = \begin{cases}\boldsymbol{f}_{\mathrm{DS} 2}\left(\boldsymbol{x}_{\boldsymbol{r}}\right) \cdot v_{d} & \text { if } \gamma<0 \\
\boldsymbol{n}\left(\boldsymbol{x}_{\boldsymbol{r}}\right) \cdot v_{d} & \text { if } \gamma>0\end{cases} \\
\gamma & =\boldsymbol{n}\left(\boldsymbol{x}_{\boldsymbol{r}}\right)\left(\boldsymbol{x}_{\boldsymbol{r}}-\boldsymbol{x}_{\boldsymbol{r}}{ }^{*}+\epsilon \cdot \boldsymbol{n}\left(\boldsymbol{x}_{\boldsymbol{r}}\right)\right)
\end{aligned}
$$

where the normal vector $\boldsymbol{n}\left(\boldsymbol{x}_{\boldsymbol{r}}\right)$ is vertical with and points to the tissue surface that is already known from demonstration data. $\gamma$ is the switch condition and $\epsilon$ is a small value for making sure the speed model changes a little before arriving at the target. When the robot does not contact the tissue, the interaction force closes to zero, which reaches a high velocity, based on the forcevelocity model $f_{\mathrm{gmr} V} V$. When the interaction force increases, the velocity magnitude decreases and the impedance gain increases based on the feedback force to make sure the robot has stable contact as shown in Fig. 5.

At the same time that the contact position is changed based on the contact force, the input position for DS1 should also be changed to make sure the motion for phase 2 is corrected. The error between the contact point and phase 1 target-point is

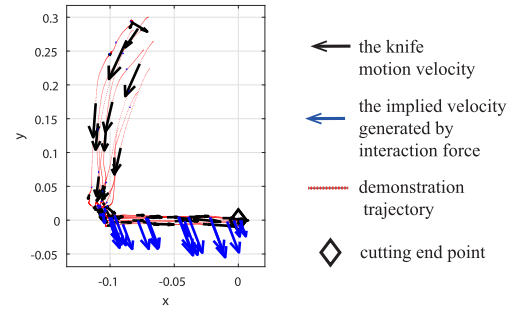

Fig. 6. There are two kinds of velocity that can be extracted from the demonstrations, real velocity and implied velocity; the latter generates a vertical interaction force.

$\boldsymbol{e}_{\text {target }}=\boldsymbol{x}_{\text {contact }}-\boldsymbol{x}_{\boldsymbol{r}}{ }^{*}$. Hence, the input robot state in $\boldsymbol{F}_{\boldsymbol{d}}\left(\boldsymbol{x}_{\boldsymbol{r}}\right)$ and $f_{\mathrm{DS} 1}\left(x_{r}\right)$ will be $\boldsymbol{x}_{\boldsymbol{r}}=\boldsymbol{x}_{\boldsymbol{r}}-\boldsymbol{e}_{\text {target. }}$. The IO model-based VA strategy modifies the coupled DS in the "motion model," and we use $f_{i}\left(x_{r}, F_{r}\right)$ to refer to it.

\section{Learning-Based DS-Modulation Strategy and FFBVI Controller}

1) DS-Modulation Strategy: When the robot cuts tissue, we not only want the robot to continue cutting the tissue, along the direction parallel to the tissue plane, but we also want the knife to maintain contact with the tissue and to make sure the knife can cut completely through the tissue.

Due to the hindrance of physical space, the movement information of the human arm is transformed from explicit information into implicit information. For example, the speed intention of arm movement is transformed into interactive force, due to physical interaction that does not show in kinematic levels, as shown in Fig. 6. This information cannot be learned by the motion model. But our setup can record the interaction force and estimate the impedance of a human arm, hence, the implicit speed information can be calculated by our demonstration data.

Using the method in [39], in order to generate a suitable vertical-interaction force, we add a DS-modulation on the DS model of out tasks. And this modulation part generates a compensation velocity that is perpendicular to the cutting plane. Hence, the motion DS is

$$
\dot{x}_{d}=\dot{x}_{d_{1}}+\dot{x}_{n}=f_{i}\left(x_{r}, F_{r}\right)+f_{n}\left(x_{r}\right) .
$$

The first term is the DS motion with an IO model that can generate the desired velocity for a cutting motion. The second term is the compensation velocity that is normal for maintaining contact with the surface to be cut. Assume that the normal vector $n\left(x_{r}\right)$ is vertical with and points to the tissue surface, so $\dot{\boldsymbol{x}}_{\boldsymbol{d}_{1}}{ }^{T} \boldsymbol{n}\left(\boldsymbol{x}_{\boldsymbol{r}}\right)=0$.

In [39], the authors generate a compensation velocity based on the desired force and the fixed damping gain. In our work, to make this method more in line with human operating habits, the compensation velocity is generated from the desired force and arm impedance from human demonstrations

$$
\dot{x}_{n}=-D\left(F_{d}\right)^{-1}\left(F_{d}\left(x_{r}\right)^{T} \cdot e_{2}\right) .
$$

2) FFBVI Controller: In order to make the controller robust to a certain degree of interaction-force variation, the FFBVI 
controller is used to adjust the controller damping eigenvalue (in our case, $\left.\boldsymbol{\Lambda}=\operatorname{diag}\left[\lambda_{1} ; \lambda_{2} ; \lambda_{3}\right]\right)$ and to generate more appropriate control torque

$$
\begin{aligned}
& \lambda_{1}=a \cdot\left\|\boldsymbol{D}_{\boldsymbol{d}}\left(\boldsymbol{F}_{\boldsymbol{r}}\right) \boldsymbol{e}_{\mathbf{1}}\right\| \\
& \lambda_{2}=\lambda_{3}=a \cdot\left\|\boldsymbol{D}_{\boldsymbol{d}}\left(\boldsymbol{F}_{\boldsymbol{d}}\right) \boldsymbol{e}_{\mathbf{2}}\right\|
\end{aligned}
$$

where $a$ is a scale factor for the robot controller, which might not approach a gain as high as a human can, and for eliminating the difference in cutting materials to obtain better control effect. The $\boldsymbol{D}_{\boldsymbol{d}}(\cdot)$ and $\boldsymbol{F}_{\boldsymbol{d}}$ are the learned GPR and GMR models proposed in Section II-C1.

The $\lambda_{1}$ adapts the controller damping gain, based on the feedback cutting-force in the cutting direction; it can give not only the damping gain a good initial value with the feedback cut force but can also increase the control gain when the output cutting force is insufficient to cut the tissue, and vice versa. The $\lambda_{2}$ adapts the vertical force, according to the desired force generated from the position-force GPR model. And $\lambda_{3}$ forces the system to selectively dissipate the energy, perpendicular to the direction of the desired movement.

The $\lambda_{1}$ ensures that the robot output-force in $\boldsymbol{e}_{1}$ direction $\left(F_{c 1}\right)$ is changed based on the interaction force, which can help the robot overcome the cutting force and ensure the cutting length. However, in other directions $\left(\boldsymbol{e}_{2}, \boldsymbol{e}_{3}\right), \lambda_{2}$ and $\lambda_{3}$ ensure that the robot maintains the contact force with the human demonstration ( $F_{c 2}=F_{d}$ and $F_{c 3}$ near to zero) to ensure the cutting depth and energy dissipation. To make this point more clear, we put the (14) into (2) and show the control output in phase 2 as follows:

$$
\begin{aligned}
& F_{c 1}=a \cdot\left\|\boldsymbol{D}_{\boldsymbol{d}}\left(\boldsymbol{F}_{\boldsymbol{r}}\right) \boldsymbol{e}_{\mathbf{1}}\right\| \cdot\left(\dot{\boldsymbol{x}}_{\boldsymbol{d} \mathbf{1}}-\dot{\boldsymbol{x}}_{\boldsymbol{r}}\right) \\
& F_{c 2}=a \cdot\left\|\boldsymbol{D}_{\boldsymbol{d}}\left(\boldsymbol{F}_{\boldsymbol{d}}\right) \boldsymbol{e}_{\mathbf{2}}\right\| \dot{\boldsymbol{x}}_{\boldsymbol{n}}=-a \cdot \boldsymbol{F}_{\boldsymbol{d}}^{\boldsymbol{T}} \boldsymbol{e}_{\mathbf{2}}
\end{aligned}
$$

where we need two feedback signals: robot real velocity $\dot{\boldsymbol{x}}_{\boldsymbol{r}}$ and interaction force $\boldsymbol{F}_{\boldsymbol{r}}$ that can be recorded by the robot operation system and force/torque sensor. For safety reasons, we limit the controller's velocity and damping gain. The upper limit of speed is set to $0.2 \mathrm{~m} / \mathrm{s}$, and the upper limit of the damping parameter is set to 160 . With these two limitations, the interaction force generated by the robot will not exceed $30 \mathrm{~N}$.

\section{E. Stability Analysis}

Passivity can be ensured if the $f(x)$ can be represented by the sum of a conservative system $\boldsymbol{f}_{\boldsymbol{c}}(\boldsymbol{x})$ with an associated potential function $\boldsymbol{V}_{\boldsymbol{f}}(\boldsymbol{x})$ (which means $\boldsymbol{f}_{\boldsymbol{c}}(\boldsymbol{x})=-\nabla \boldsymbol{V}_{\boldsymbol{f}}(\boldsymbol{x})$ ), a nonconservative system $\boldsymbol{f}_{\boldsymbol{r}}(\boldsymbol{x})$, and a compensation DS system $\boldsymbol{f}_{\boldsymbol{n}}(\boldsymbol{x})$ [39]

$$
\dot{x}_{d}=f(x)=f_{c}(x)+f_{r}(x)+f_{n}(x) .
$$

For the storage function $W\left(\boldsymbol{x}_{\boldsymbol{r}}, \dot{\boldsymbol{x}}_{\boldsymbol{r}}\right)=\frac{\mathbf{1}}{\mathbf{2}} \dot{\boldsymbol{x}}_{\boldsymbol{r}}^{T} \boldsymbol{M}\left(\boldsymbol{x}_{\boldsymbol{r}}\right) \dot{\boldsymbol{x}}_{\boldsymbol{r}}+$ $\lambda_{1}(t) \boldsymbol{V}_{\boldsymbol{f}}(\boldsymbol{x})$, by using the control law in (2) and virtual energy storage, we can ensure that the whole system is passive with fixed damping gain.
However, since our controller also changes the damping gain, based on the interaction force, the energy tank, proposed in [39], cannot be applied directly (as it assumed a fixed gain).

Taking into account the change of the damping over time, the time derivative of $W\left(\boldsymbol{x}_{\boldsymbol{r}}, \dot{\boldsymbol{x}}_{\boldsymbol{r}}\right)$ is

$$
\begin{aligned}
\dot{W}\left(\boldsymbol{x}_{r}, \dot{x}_{r}\right)= & \frac{1}{2} \dot{\boldsymbol{x}}_{r}^{T} \dot{M}\left(\boldsymbol{x}_{r}\right) \dot{x}_{r}+\dot{x}_{r}^{T} M\left(x_{r}\right) \ddot{x}_{r} \\
& +\lambda_{1} \nabla V_{f}\left(x_{r}\right)^{T} \dot{x}_{r}+\dot{\lambda}_{1} V_{f}(x) .
\end{aligned}
$$

The last term of the right-hand side cannot be eliminated by the conservative system $\boldsymbol{f}_{\boldsymbol{c}}(\boldsymbol{x})$ in (16).

Therefore, to control the energy-injection caused by varying the damping term, we separate the varying-damping gain into a constant term and a time-variable term, as $\lambda_{1}=\lambda_{1 C}+\lambda_{1 V}(t)$. The constant term $\lambda_{1 C}$ can remain passive by using the same energy tank: $\dot{s}=\alpha(s) z_{d}-\beta_{r}\left(s, z_{r}\right) \lambda_{1 C} z_{r}-\beta_{n}\left(s, z_{n}\right) \lambda_{1 C} z_{n}$, as in [39], and $z_{d}=\dot{\boldsymbol{x}}_{\boldsymbol{r}}^{\boldsymbol{T}} \boldsymbol{D}\left(\boldsymbol{x}_{\boldsymbol{r}}\right) \dot{\boldsymbol{x}}_{\boldsymbol{r}}, z_{r}=\dot{\boldsymbol{x}}_{\boldsymbol{r}}^{\boldsymbol{T}} \boldsymbol{f}_{\boldsymbol{r}}\left(\boldsymbol{x}_{\boldsymbol{r}}\right), z_{n}=$ $\dot{\boldsymbol{x}}_{\boldsymbol{r}}^{\boldsymbol{T}} \boldsymbol{f}_{\boldsymbol{n}}\left(\boldsymbol{x}_{\boldsymbol{r}}\right)$. For the time-varying term $\lambda_{1 V}(t)$, we add an additional energy tank coupled with the energy tank in [39]. We modify this energy-tank state $s \in \mathbb{R}$ as follows:

$$
\dot{s}=\alpha(s) z_{d}-\beta_{r}\left(s, z_{r}\right) \lambda_{1 C} z_{r}-\beta_{n}\left(s, z_{n}\right) \lambda_{1 C} z_{n}-x_{t} \dot{x}_{t}
$$

where the term $x_{t}(t) \in \mathbb{R}$ is the state associated with the tank $\left(\dot{x}_{t}=\boldsymbol{\omega}^{T} \dot{\boldsymbol{x}}_{\boldsymbol{r}}\right.$ ), and $\alpha(s), \beta_{r}, \beta_{n}, \boldsymbol{\omega}$ are the functions that control the energy flow between the energy tank and the robot.

To control the energy generated by the DS model, we modify the final DS model as follows:

$$
\boldsymbol{f}_{\boldsymbol{\beta}}(x)=\boldsymbol{f}_{\boldsymbol{c}}(\boldsymbol{x})+\beta_{r}{ }^{\prime}\left(s, z_{r}\right) \boldsymbol{f}_{\boldsymbol{r}}(\boldsymbol{x})+\beta_{n}{ }^{\prime}\left(s, z_{n}\right) \boldsymbol{f}_{\boldsymbol{n}}(\boldsymbol{x})
$$

We set the scalar functions $\alpha(s), \beta_{r}, \beta_{n}, \beta_{r}{ }^{\prime} \beta_{n}{ }^{\prime}$ same with [39] as shown in (27)-(29) and set the $\boldsymbol{\omega}$ as

$$
\boldsymbol{\omega}(t)= \begin{cases}\frac{\lambda_{1 V}(t) \boldsymbol{f}_{\boldsymbol{\beta}}\left(\boldsymbol{x}_{\boldsymbol{r}}\right)}{x_{t}} & \text { if } s_{m}>s>s_{m}-\theta_{s} \\ 0 & \text { otherwise }\end{cases}
$$

where $s_{m}-\theta_{s}$ is a threshold below which the energy cannot be extracted by the tank; $s_{m}$ is the maximum energy level allowed us to be stored in the tank. And to avoid singularities, we set $x_{t}(0)>0$ and $x_{t}(t)$ be a very small positive value if it equal to zero.

To take the state of the tank and the power variables into account, we corrected the control law in (2) and using the corrected motion generator in (19) as follows:

$$
\boldsymbol{F}_{\boldsymbol{c}}=-\boldsymbol{D}\left(\boldsymbol{x}_{\boldsymbol{r}}\right) \dot{\boldsymbol{x}}_{\boldsymbol{r}}+\lambda_{1 C} \boldsymbol{f}_{\boldsymbol{\beta}}\left(\boldsymbol{x}_{\boldsymbol{r}}\right)+\boldsymbol{\omega} x_{t} .
$$

With all abovementioned setting, we can ensure that the whole system is passive. Now we will describe the proof of the controller's passivity based on our improved method.

Proof: We consider the storage function $W\left(\boldsymbol{x}_{\boldsymbol{r}}, \dot{\boldsymbol{x}}_{\boldsymbol{r}}\right)=$ $\frac{\mathbf{1}}{\mathbf{2}} \dot{\boldsymbol{x}}_{\boldsymbol{r}}^{\boldsymbol{T}} \boldsymbol{M}\left(\boldsymbol{x}_{\boldsymbol{r}}\right) \dot{\boldsymbol{x}}_{\boldsymbol{r}}+\lambda_{1 C} \boldsymbol{V}_{\boldsymbol{f}}(\boldsymbol{x})+s, \quad$ in which $\lambda_{1}=\lambda_{1 C}+$ $\lambda_{1 V}(t)$. So the time derivative of $W\left(\boldsymbol{x}_{\boldsymbol{r}}, \dot{\boldsymbol{x}}_{\boldsymbol{r}}\right)$ is

$$
\begin{aligned}
\dot{W}\left(\boldsymbol{x}_{r}, \dot{\boldsymbol{x}}_{\boldsymbol{r}}\right)= & \dot{\boldsymbol{x}}_{\boldsymbol{r}}^{\boldsymbol{T}} M\left(\boldsymbol{x}_{\boldsymbol{r}}\right) \ddot{\boldsymbol{x}}_{\boldsymbol{r}}+\frac{1}{2} \dot{\boldsymbol{x}}_{\boldsymbol{r}}^{\boldsymbol{T}} \dot{M}\left(\boldsymbol{x}_{\boldsymbol{r}}\right) \dot{\boldsymbol{x}}_{\boldsymbol{r}} \\
& +\lambda_{1 C} \nabla \boldsymbol{V}_{\boldsymbol{f}}\left(\boldsymbol{x}_{\boldsymbol{r}}\right)^{\boldsymbol{T}} \dot{\boldsymbol{x}}_{\boldsymbol{r}}+\dot{s} .
\end{aligned}
$$


Substituting (1) for $\boldsymbol{M}\left(\boldsymbol{x}_{\boldsymbol{r}}\right) \ddot{\boldsymbol{x}}_{\boldsymbol{r}}$ and (21) for $\boldsymbol{F}_{\boldsymbol{c}}$ yields

$$
\begin{aligned}
\dot{W}\left(x_{r}, \dot{x}_{r}\right)= & \dot{x}_{r}^{T}\left(-C\left(\dot{x}_{r}, x_{r}\right) \dot{x}_{r}-D\left(x_{r}\right) \dot{x}_{r}\right. \\
& \left.+\lambda_{1 C} f_{\beta}\left(x_{r}\right)+\omega x_{t}+F_{e}\right) \\
& +\frac{1}{2} \dot{x}_{r}^{T} \dot{M}\left(x_{r}\right) \dot{x}_{r}+\lambda_{1 C} \nabla V_{f}\left(x_{r}\right)^{T} \dot{x}_{r}+\dot{s}
\end{aligned}
$$

Using the fact that $\dot{M}-\mathbf{2 C}$ is skew symmetric and that $f_{c}(x)=-\nabla V_{f}(x)$ (by construction) and (19), then we rearrange the formula:

$$
\begin{aligned}
\dot{W}\left(\boldsymbol{x}_{\boldsymbol{r}}, \dot{\boldsymbol{x}}_{\boldsymbol{r}}\right)= & \beta_{r}{ }^{\prime}\left(s, z_{r}\right) \dot{\boldsymbol{x}}_{\boldsymbol{r}}^{\boldsymbol{T}} \lambda_{1 C} \dot{\boldsymbol{f}}_{r}+\beta_{n}{ }^{\prime}\left(s, z_{n}\right) \dot{\boldsymbol{x}}_{\boldsymbol{r}}^{\boldsymbol{T}} \lambda_{1 C} \dot{\boldsymbol{f}}_{n} \\
& +\dot{\boldsymbol{x}}_{\boldsymbol{r}}^{\boldsymbol{T}} \boldsymbol{\omega} x_{t}-\dot{\boldsymbol{x}}_{\boldsymbol{r}}^{\boldsymbol{T}} \boldsymbol{D}\left(\boldsymbol{x}_{\boldsymbol{r}}\right) \dot{\boldsymbol{x}}_{\boldsymbol{r}}+\dot{\boldsymbol{x}}_{\boldsymbol{r}}^{T} \boldsymbol{F}_{\boldsymbol{e}}+\dot{s}
\end{aligned}
$$

The fourth and fifth terms in (24) show that the system is passive with respect to the input/output pair of $\boldsymbol{F}_{e}, \dot{\boldsymbol{x}}_{r}$. To stabilize the system, we add three terms for the energy tank to control the undefined first three terms of (24). Inserting the terms of (18) into (24) we get

$$
\begin{aligned}
\dot{W}\left(\boldsymbol{x}_{\boldsymbol{r}}, \dot{\boldsymbol{x}}_{\boldsymbol{r}}\right)= & \beta_{r}{ }^{\prime}\left(s, z_{r}\right) \dot{\boldsymbol{x}}_{\boldsymbol{r}}^{\boldsymbol{T}} \lambda_{1 C} \dot{\boldsymbol{f}}_{\boldsymbol{r}}+\beta_{n}{ }^{\prime}\left(s, z_{n}\right) \dot{\boldsymbol{x}}_{\boldsymbol{r}}^{T} \lambda_{1 C} \dot{\boldsymbol{f}}_{\boldsymbol{n}} \\
& +\dot{\boldsymbol{x}}_{\boldsymbol{r}}^{\boldsymbol{T}} \boldsymbol{\omega} x_{t}-\dot{\boldsymbol{x}}_{\boldsymbol{r}}^{\boldsymbol{T}} \boldsymbol{D}\left(\boldsymbol{x}_{\boldsymbol{r}}\right) \dot{\boldsymbol{x}}_{\boldsymbol{r}}+\dot{\boldsymbol{x}}_{\boldsymbol{r}}^{T} \boldsymbol{F}_{\boldsymbol{e}} \\
& -\beta_{r}\left(s, z_{r}\right) \lambda_{1 C} z_{r}-\beta_{n}\left(s, z_{n}\right) \lambda_{1 C} z_{n} \\
& -x_{t} \dot{x}_{t}+\alpha(s) z_{d} .
\end{aligned}
$$

Substituting that $\dot{x}_{t}=\boldsymbol{\omega}^{T} \dot{\boldsymbol{x}}_{\boldsymbol{r}}$ yields

$$
\begin{aligned}
\dot{W}\left(\boldsymbol{x}_{\boldsymbol{r}}, \dot{\boldsymbol{x}}_{\boldsymbol{r}}\right)= & -(1-\alpha(s)) z_{d}-\left(\beta_{r}\left(s, z_{r}\right)-\beta_{r}{ }^{\prime}\left(s, z_{r}\right)\right) \lambda_{1 C} z_{r} \\
& +\dot{\boldsymbol{x}}_{\boldsymbol{r}}^{\boldsymbol{T}} \boldsymbol{F}_{\boldsymbol{e}}-\left(\beta_{n}\left(s, z_{n}\right)-\beta_{n}{ }^{\prime}\left(s, z_{n}\right)\right) \lambda_{1 C} z_{n} .
\end{aligned}
$$

Using the same set of $\alpha(s), \beta_{r}, \beta_{n}, \beta_{r},{ }^{\prime} \beta_{n}{ }^{\prime}$ as shown in [39]

$$
\alpha(s)= \begin{cases}1 & s<s_{m}-\theta_{s} \\ \frac{1}{2}\left(1+\cos \left(\pi \frac{s-\left(s_{m}-\theta_{s}\right)}{s_{m}-\left(s_{m}-\theta_{s}\right)}\right)\right) & s_{m}-\theta_{s} \leqslant s \leqslant s_{m} \\ 0 & s>s_{m}\end{cases}
$$

$\beta_{i}\left(s, z_{i}\right)=\left\{\begin{array}{l}0 \text { if } s<0 \text { and } z_{i}>0 \\ 0 \text { if } s>s_{m} \text { and } z_{i}<0 \quad i=r, n \\ 1 \text { otherwise }\end{array}\right.$

$\beta_{i}{ }^{\prime}\left(s, z_{i}\right)=\left\{\begin{array}{ll}1 & \text { if } z_{i}<0 \\ \beta_{i}\left(s, z_{i}\right) & \text { otherwise }\end{array} \quad i=r, n\right.$

we ensure the following:

1) The first, second, and last terms of (26) are under the control of energy tank and will remain negative or zero at all times, hence ensuring that the system is passive with respect to the input-output pair $\dot{\boldsymbol{x}}_{r}^{T} \boldsymbol{F}_{\boldsymbol{e}}$.

2) The energy injected in the tank equals the energy injected by the mechanical impedance.

3 ) If the energy tank is depleted, the $\beta_{i}{ }^{\prime}\left(s, z_{i}\right)$ terms can be used to modify the control input and bound the energy injected by our dynamical system $f_{i}(i=r, n)$; and the energy generated by variable damping gain is under

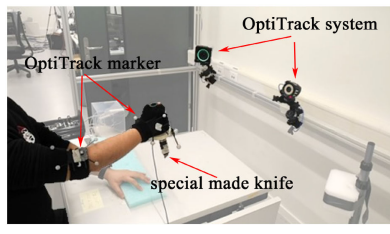

(a)

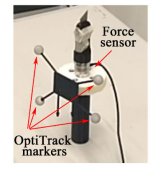

(b)
Fig. 7. Data recording setup. Position of knife and hand are tracked via Optitrack markers. Forces are measured at the tip of the tool held in hand, with nano ATI sensor. EMG data is recorded to monitor changes in arm impedance.

control of $\boldsymbol{\omega}$, the desired variable-damping gain will be implemented or not implemented based on the state of energy tank.

Hence, based on the set of the energy tank, the system is passive.

\section{EXPERIMENTS AND RESULTS}

To validate the method, we showcase that we can extract the variables from human demonstration and then use these for control. Furthermore, we show that our control method can generalize cutting to different tissues.

\section{A. Record and Analysis of Human Cutting-Data}

To record data from human demonstration, the same subject (here, the experimenter) was tasked to make a linear incision 9 times on the same type of off-the-shelf plastic. To prevent damage to the force sensor, we chose this material specifically to ensure that the maximum cutting force in the actual cutting process is lower than the safety threshold of the force sensor. And to train a more generalized model, we also need to ensure that the tissue can provide a sufficiently large range of cutting force to cover the force needed by the robot-cut tissue. Note that though we train on plastic, we test the system on silicon, see the following section. The choice of using different material for training and testing is meant to demonstrate that we learn a skill to cut soft material but not specifically for one type of material.

Each cut starts from the same location and has a similar length (about $20 \mathrm{~cm}$ ). An ATI Nano17 sensor mounted on the tool is used for recording the interaction force between hand and knife, at $100 \mathrm{~Hz}$ (see Fig. 7). An OptiTrack motion-capture system $(100 \mathrm{~Hz})$ with markers attached to the tool, to the human hand and to the elbow, is used for recording the position data. The EMG data of flexor carpiulnaris on human forearm is recorded by TI ADS1298 bio-signal amplifiers $(1000 \mathrm{~Hz})$. To obtain the estimation of human-arm impedance, we apply a similar method, as described in [38]. The arm position and EMG signal, we collected are processed as the input of a conversion function; and the output of this function is the arm end-point impedance. The parameters of this function is calculated by the least squares method by using the data and results of a human-arm impedance identification experiment. The Butterworth low-pass filter was used to filter the position and force data with cut-off frequency of $18 \mathrm{~Hz}, 12 \mathrm{~Hz}$. 


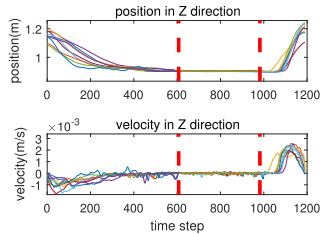

(a)
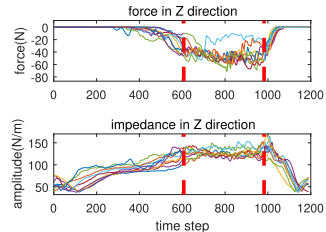

(b)
Fig. 8. Human demonstration data. The curve is the demonstration data that was divided into three phases; and the red dotted line is the dividing line of the different phases.


Fig. 9. Learned modulation of the velocity. We chose a random demonstration trial of data (only phase 1) as the input (force) and ground truth (velocity) of the model regression. Left figure: the force recorded in the demonstration increased when the knife contacted with the tissue (around 350 time steps). Right figure: The regression result velocity of the force-velocity model followed the change of input force and did so similarly with the ground truth velocity.

The human demonstration data are illustrated in Fig. 8. We divide the cutting task into three phases and align data for all three phases ( 9 trials and a total of 7904 data points: 3338, 2251, and 1505 data points for phase 1,2 , and 3). Then, three phases of demonstration data were separated into training sets $(60 \%)$ and test sets $(40 \%)$ for the position-force model and the forceimpedance model. The phase 1 data was used to train the forcevelocity model, with the same proportion of the training-test set. The position and velocity data in each phase were used for learning the corresponding DSs in coupled DS model. In our work, 10-fold cross-validation and Bayesian information criterion are performed to find the best number of Gaussians for the GMM-GMR model (4 for position-force model and 3 for force-velocity model); the DS model is learned by the SEDS algorithm [32]; and the force-impedance model is learning by the GPR algorithm [40]. The learning and test results of these models are partly shown in Fig. 9 (force-velocity model) and 10 (force-impedance model).

\section{B. Performance of the Robot Cutting-Task}

Once the model was successfully trained offline, we conducted two groups of cutting experiments by using our 7-DOF KUKA light-weight robot arm. The setup of this experiment is shown in Fig. 11. The robot is controlled through a fast research interface (FRI) that can provide the communication between the controlling computer and the control box. Through the FRI, we receive the robot-state feedback and send the control commands.



Fig. 10. Upper figure: The GPR model (regression and variance) learned to embed a model of the variance of impedance as a function of the interaction force. Lower figure: Using a random trial of data as input (force) and ground truth (impedance) to test the GPR model, the impedance increased around 400 time steps with the force increased, then it decreased at 900 time steps as the force disappeared.

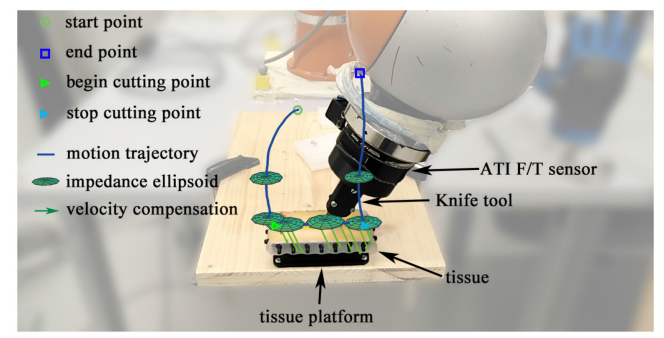

Fig. 11. Robot cutting-task. A knife tool is fixed on an ATI F/T sensor and installed at the end of robot. The silicone tissue is attached to a platform to ensure that it is stretched to reduce cutting resistance [14]. During the robot action, our algorithm used models previously learned in order to calculate all the control output, such as the impedance and velocity.

An ATI F/T sensor (Gamma) is used to collect the feedback interaction force. The purpose of the experiment was three-fold:

1) To demonstrate the performance of the closed-loop approach, as compared to using impedance-based control with fixed and variable impedance.

2) To assess the ability of the learned controller to generalize and adapt to different tissues and to different starting conditions.

3) To verify the response of coupled DS to different kinds of disturbances.

We used artificial tissues made of silicon [41] that bears a close resemblance, in its elastic texture, to the human skin of the human body. ${ }^{2}$

Robustness to tissue's change in hardness. In the first group of experiments, we contrasted our approach (FFBVI controller) with a fixed impedance and a position-based variable-impedance controller. To assess the role played by each part of our control system on performance, the VA strategy and DS-modulation strategy, respectively, are at times used or omitted.

A total of five experiments were carried out; these performance are presented in Table I. For each experimental condition,

\footnotetext{
${ }^{2}$ We ensured that the shape and thickness of the tissue were the same by pouring the silica gel solution into the mold; and we ensured that the hardness of the silicon tissue was different, by purchasing the solutions with different hardness parameters.
} 
TABLE I

COMPARISON OF PERFORMANCE OF ROBOt CUTTING-EXPERIMENT With DIFFERENT STRATEGIES

\begin{tabular}{lcccc}
\hline $\begin{array}{l}\text { Exp } \\
\text { name }\end{array}$ & $\begin{array}{c}\text { average cut } \\
\text { depth (\%) }\end{array}$ & $\begin{array}{c}\text { average cut } \\
\text { length (\%) }\end{array}$ & $\begin{array}{c}\text { max contact } \\
\text { peak force (N) }\end{array}$ & $\begin{array}{c}\text { success } \\
\text { rate (\%) }\end{array}$ \\
\hline Exp 1. & 81.11 & 65.56 & 14.4 & 11.1 \\
\hline Exp 2. & 81.25 & 90 & 11.7 & 37.5 \\
\hline Exp 3. & 86.7 & 100 & 23.6 & 66.7 \\
\hline Exp 4. & 100 & 100 & 24.5 & 100 \\
\hline Exp 5. & 100 & 100 & 11.6 & 100 \\
\hline
\end{tabular}

Exp 1: fixed impedance controller; Exp 2: position based impedance controller; Exp 3: FFBVI controller; Exp 4: Exp 3 add DS-modulation strategy; Exp 5: Exp 4 add VA strategy for phase 1 .

six trials were conducted (each trial performs on one tissue). To assess performances against the tissue's hardness, two kinds of tissues were used in Experiments 3 and 5. Three tissues were a hardness of 30 and others were a hardness of 50 . We recorded the interaction force, knife position, and the impedance controller gains.

To evaluate our method, we compared the results in terms of success rate, depth of cut, length of cut, and the maximum force during penetration. By measuring and calculating the percentage of the cut marks that correspond to the tissue thickness, we calculated the depth of the cut for each trial. And the length of the cut was calculated by comparing the actual length that was cut to the expected length in the DS1 model. If both the length and depth of the cut were $100 \%$, we called it a successful cutting trial. If the knife was stuck and the length cut is insufficient (lower than $100 \%$ ) or the knife did not cut through the tissue (depth of cut was lower than $100 \%$ ), we defined it as a failed trial. In this situation, we use the shallowest cut as the cutting depth, and the length of the part that was cut completely through as the cutting length. The maximum interaction force in the direction perpendicular to the contact surface in the approach phase is recorded as the maximum force during penetration.

As shown in Table I, the success rates were low in Exp. 1-3, when using the nonadaptive controllers. The robot was stopped most frequently by high interaction force that came from the cutting process (average cutting length was not 100\%) or it could not cut through the tissue, due to the upward interaction force (average depth of the cuts was not 100\%). In these situations, the highest interaction force peak was observed when the knife entered the tissue, which not only made it difficult for the robot to overcome the cutting force, but also might cause unexpected tissue damage. In contrast, when using our adaptive method (Exp 4 and 5), the success rate of the task increased substantially, and the cutting depth and average cutting length could reach $100 \%$.

These results confirm that the force-based controller has interactive-adjustment capabilities better than the fixed impedance or position-based variable impedance controllers. It could ensure that the robot was not blocked by an unexpected interaction force. The DS-modulation strategy contributed to ensuring that the cutting was completed in its entirety hence that the depth and length of the cut were close to the expected
TABLE ॥

Performance For DifFERENT TISSUE'S haRdNesS

\begin{tabular}{cccccc}
\hline \multicolumn{6}{c}{ Exp 5. Exp 4 add VA strategy for phase 1 } \\
\hline trials & $\begin{array}{c}\text { tissues } \\
\text { hardness }\end{array}$ & $\begin{array}{c}\text { max contact } \\
\text { peak } \\
\text { force }(\mathrm{N})\end{array}$ & $\begin{array}{c}\text { average } \\
\text { contact } \\
\text { force }(\mathrm{N})\end{array}$ & $\begin{array}{c}\text { cut } \\
\text { length } \\
(\%)\end{array}$ & $\begin{array}{c}\text { cut } \\
\text { depth } \\
(\%)\end{array}$ \\
\hline 1 & 30 & 11.7 & $3.18 \pm 1.35$ & 100 & 100 \\
2 & 30 & 8.5 & $3.13 \pm 1.42$ & 100 & 100 \\
3 & 30 & 5.5 & $2.84 \pm 0.82$ & 100 & 100 \\
4 & 50 & 7.2 & $2.99 \pm 1.24$ & 100 & 100 \\
5 & 50 & 7 & $2.05 \pm 1.58$ & 100 & 100 \\
6 & 50 & 7 & $3.69 \pm 1.18$ & 100 & 100 \\
\hline \multicolumn{5}{c}{ Exp 3. FFBVI controller } \\
trials & hardness & max contact & average & cut & cut \\
& peak & contact & length & depth \\
1 & 30 & 6.8 & $2.05 \pm 1.66$ & 100 & 100 \\
2 & 30 & 6.7 & $1.97 \pm 1.68$ & 100 & 100 \\
3 & 30 & 5.4 & $1.57 \pm 1.21$ & 100 & 100 \\
4 & 50 & 17.8 & $2.60 \pm 3.09$ & 100 & 80 \\
5 & 50 & 17.5 & $2.33 \pm 2.63$ & 100 & 40 \\
6 & 50 & 23.6 & $2.36 \pm 2.69$ & 100 & 100 \\
\hline
\end{tabular}

percentage. Credit goes to the VA strategy, as the peak of the interaction force in phase 1 also decreased and became controllable, which means the execution of the cut is safer and better.

Table II compares performance depending on the tissues' hardness. To quantify the interaction, we measure the average contact force and report also the standard deviation (STD); we compute the interaction force perpendicular to the cutting direction in the cutting phase. The experiments recorded in Table II are also the Exp 3. and Exp 5. in Table I. We can see with our VA and DS-modulation strategies that the cutting operation had a high success rate. And for the two kinds of tissues, the length and depth of the cut remain at $100 \%$, the max-contact peak force is relatively small and limited to a smaller range of changes, the average contact force is kept within a certain range, and the deviation of the force is relatively small.

Robustness to changes in the tissue's configuration. In a second group of experiments, we qualitatively analyzed the robustness of our proposed model and the controller (same setup as Exp 5. in Table I) for different experimental configurations. We focused on establishing the controllers' ability (or inability) to adapt to changes in the experimental conditions. To generate unexpected changes while cutting, a human subject would interrupt the robot during the motion. To guarantee that the task was safe for the subject, we replaced the knife with a smooth cylinder.

1) Disturbances Prior to Execution: To compare the IO model's and the VA strategy's abilities to respond to position error in the vertical direction (typically when the contact between the knife and the tissue occurs at an unexpected height), we conducted 18 trials where we modified the position of the tissue in the $Z$ direction: 6 trials were run with a tissue located $25 \mathrm{~mm}$ higher than the desired contact position. Six trials were run with the tissue in the desired contact position. The last 6 trials were run with a tissue $25 \mathrm{~mm}$ lower than desired position. The results are shown in Table III. 
TABLE III

Results of Cutting Under Different Contact Positions and With/Without the VA Strategy Assistance

\begin{tabular}{|c|c|c|c|c|c|c|c|c|c|c|c|c|c|c|}
\hline \multicolumn{7}{|c|}{ maximum contact peak force $(\mathrm{N})$ with strategy assistance } & std & \multicolumn{6}{|c|}{ force $(\mathrm{N})$ without strategy assistance } & \multirow[t]{2}{*}{ std } \\
\hline expected position & 8.69 & 9.22 & 8.8 & 8.9 & 8.2 & 8.08 & & 1.94 & fail & 2.6 & 1.45 & 2.15 & fail & \\
\hline $25 \mathrm{~mm}$ higher & 4.15 & 6.85 & 5.12 & 5.65 & 4.35 & 1.93 & +240 & 15 & 15.9 & 20.15 & 18.2 & 21.4 & 20.4 & +873 \\
\hline $25 \mathrm{~mm}$ lower & 4.7 & 4.5 & 2.46 & 5.27 & 4.04 & 2.7 & & fail & fail & fail & fail & fail & fail & \\
\hline
\end{tabular}
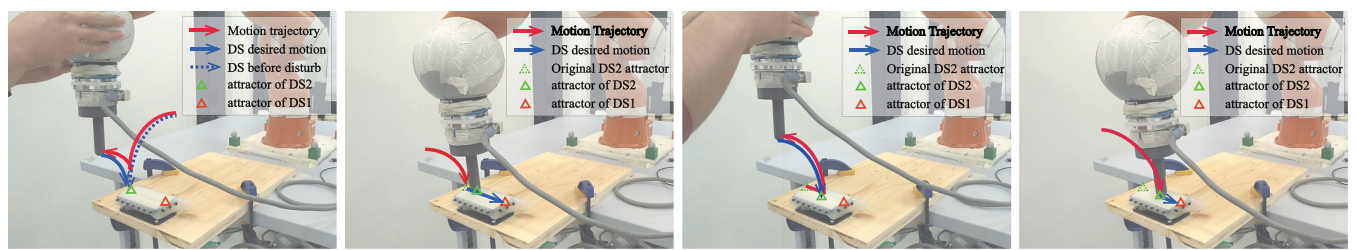

Fig. 12. From left to right, these 4 figures show 1) robot approaches (red arrow line) the beginning cutting point (Green triangle) follows the DS1 (blue dotted line), when it get disturb, DS1 will replan the trajectory (blue line), 2) robot returns to disturbance point under control of DS2 and begins cutting under control of DS1, while the attractor of DS2 will follow the motion of robot, 3) robot is disturbed during cutting, so DS2 is actived again and replan the trajectory to go back to the disturbance point, and 4) robot returns to disturbance point and continues cutting.

2) Disturbances during execution: To test the ability of our proposed coupled DS model and the controller to handle disturbances generated by a human during task execution, we generated disturbances during different phases of motion. We observed how the robot responded to the disturbances. All these experiments are shown in the accompanying Video. ${ }^{3}$ Snapshots are visible in Fig. 12.

Table III shows results with and without the VA strategy assistance, when adapting to errors in the expected vertical positions of the tissue. Observe that, without the assistance of our proposed adaptive controller, the task failed many times. The peak force at contact changed significantly (from 1.45 to $21.4 \mathrm{~N}$ ) under different experiment configurations. This is due to the fact that the speed generated by the SEDS model is not related to the contact situation, rather it is related to the desired contact position (as shown in Fig. 5). When the contact occurs earlier than the expected position, it will cause a high contact force under high SEDS velocity. For the situation where the real contact point is lower than desired, the motion of SEDS will stop and be unable to make contact. Furthermore, the inaccuracy of the compliant control system also make it hard to find an ideal contract point. Consequently, for different trials, the force data will change substantially. This phenomenon is also reflected in Table II, the maximum contact peak force changes significantly under different trials, and the STD of an average contact force is larger than when using strategy assistance.

With the assistance of the adaptive strategy, all the tasks were successful. The change of the maximum force during penetration was also controlled within a certain range (4.04 to $9.22 \mathrm{~N}$ ), which means that in the interaction of uncertain contact positions, our strategy reduces the change of the maximum contact force. Using the data in Table III to calculate the STD (not considering failed tasks) of the maximum force during penetration, our adaptive strategy can reduce this value from $\pm 8.73 \mathrm{~N}$ to $\pm 2.4 \mathrm{~N}$. Similar results can be seen in Table II.

${ }^{3}$ [Online]. Available: https://youtu.be/KAOxU7RhLjg
Our subsequent experiment tested the response of our algorithm to disturbances that occurred "live." When the robot was disturbed during phase 1 , the robot was being run under the control of DS2, hence, the robot changed its motion trajectory by basing its calculation on the SEDS model, thus driving the robot's approach to the target point. In phase 2, DS1 was activated and it controlled the cutting. When the robot was disturbed, the DS2 became active again and pulled the robot back to the beginning point of the disturbance. The whole process is shown in Fig. 12.

The real-time changes of impedance and force, during the robot's operation, can also reflect the adaptability of the algorithm in dealing with uncertain factors. In another experiment, except for interrupting the robot cutting, we also artificially increased the cutting force (used a hand to obstruct the cutting motion), and we observed the controller's response to the change of the interactive force. To ensure that the cutting can be completed, the FFBVI controller changes the control gain during cutting. The energy tank also guarantees the passivity of the system by storing and releasing energy during the interaction. As shown in Fig. 13, to help the robot to overcome the cutting force and to keep passivity during interaction, the eigenvalue 1 and $2\left(\lambda_{1}, \lambda_{2}\right)$ of the damping $D$ matrix change based on the interaction force, the state of energy tank, and the direction of motion.

\section{SUMMARY AND DISCUSSION}

Our framework for learning variable-impedance cutting skills from humans was tested on a robot automatic-cutting experiment. To validate the importance of closing the loop in force and of adapting the impedance from force-feedback, we contrasted our approach to a fixed impedance and a position-based variable-impedance controller. We shown that with our approach the robot can execute the cutting with a high success rate and can adapt to different task conditions. Also, when the robot is disturbed during different motion states, it can automatically return to the breaking point with different reactions. 


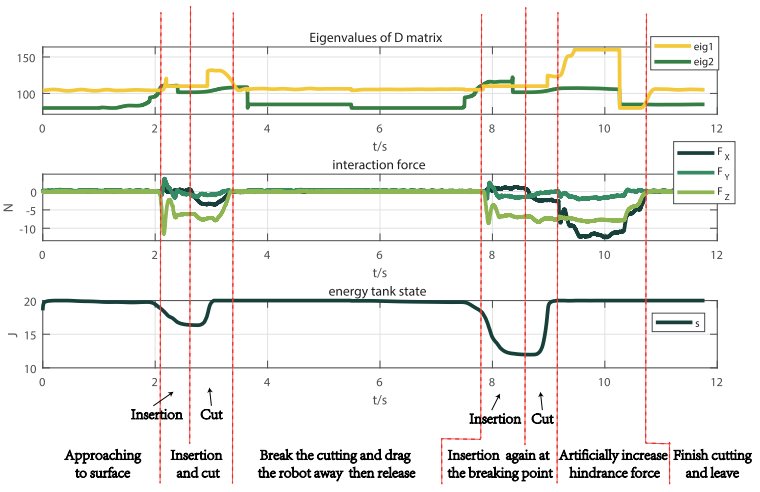

Fig. 13. Modulation of damping gain (up) based on force feedback (middle) and state of energy tank (below) during a real-robot automatic cutting. The red dashed line is used to separate the different operating phases. During the "insertion and cut" phase, the damping eigen-value showed an increase based on the $Z$-direction insertion force. And during the "artificially increased hindrance force" phase, the eig1 showed a significant increase based on the $X$-direction cutting force. To perform these nonpassive actions, the system extracted energy from the energy tank, during the penetration phase and the initial stage of cutting.

The VA strategy can automatically process the motion error caused by the inaccuracy of the robot's motion and due to unknown tissue thickness, and it can adjust the learning model so that the robot can smoothly switch from insertion to cutting and can ensure the success of insertion. The uncertain contact force during insertion, which could cause unexpected tissue damage, is now controllable.

We extend the DS-modulation strategy [39] to modulate the impedance. Specifically, we combined this strategy with our learning-based IO model to achieve a desired interaction force for the whole task. This strategy can maintain the knife in a controllable interaction force in a nonprimary movement direction, while the IO model can control the force in cutting direction. From the point of view of human-arm operation mechanism, the strategy is clearly observed and verified in the demonstration data we recorded. Hence, we learned a more anthropomorphic interaction strategy from humans. We show that we learned both the interaction force and impedance gain, directly from the human demonstrations, and that we applied them to the robot's task, and that this shows a good performance.

Stability comes from two levels, the first level is that we have a stable DS motion generator [32], the SEDS can generate a trajectory in real time; it converges to the target in the absence of disturbance. Whereas, at the second level, with disturbances and interactions, we have an energy-tank method to ensure the passivity of the whole system. The stability we provide is different for [28] ensuring the controller convergence to tracking trajectory. This is caused by the characteristics (real-time motion generator) and the needs (human-robot interaction) of our method.

When we compare our work with the work in [29], we do not have a safety mechanism to prevent the robot from exceeding a certain workspace, which might cause danger for the human and the environment around the robot. Although the coupled DS model can ensure the trajectory of robot can converges to the target point and return to the break-point after disturbance, the workspace for these two DS models still needs a safety limitation.

\section{LIMITATION AND FUTURE WORK}

We summarize the limitations of the current work as follows:

1) The method was only tested to cut linear trajectories.

2) Some of the controller gains still need to be adjusted to fit the differences in impedance parameters between the human arm and the robot.

3) The generalization ability for objects of different physical properties needs to be improved.

4) To prevent the robot from exceeding a certain workspace, a safety mechanism is needed.

These limitations, however, can be addressed in our future work by learning from the different physical properties of tissue-cutting demonstrations and different nonlinear cuttingtrajectory demonstrations. To teach the robots how to adjust their impedance parameters for new kinds of tissues, based on their sensor system and human demonstrations, would be an interesting research direction.

\section{ACKNOWLEDGMENT}

The authors would like to thank the China Scholarship Council and Zeiss for their financial support. The authors also would like to thank N. Figueroa, M. Khoramshahi, W. Amanhoud, L. Huber, K. Yao, and S. M. Hadi Sadati for their help and assistance with this article.

\section{REFERENCES}

[1] F. J. Abu-Dakka, B. Nemec, J. A. Jørgensen, T. R. Savarimuthu, N. Krüger, and A. Ude, "Adaptation of manipulation skills in physical contact with the environment to reference force profiles," Auton. Robots, vol. 39, no. 2, pp. 199-217, 2015.

[2] F. Suárez-Ruiz and Q.-C. Pham, "A framework for fine robotic assembly," in Proc. IEEE Int. Conf. Robot. Autom., 2016, pp. 421-426.

[3] N. Figueroa, A. L. P. Ureche, and A. Billard, "Learning complex sequential tasks from demonstration: A pizza dough rolling case study," in Proc. 11th ACM/IEEE Int. Conf. Hum.-Robot Interact., 2016, pp. 611-612.

[4] L. P. Ureche and A. Billard, "Constraints extraction from asymmetrical bimanual tasks and their use in coordinated behavior," Robot. Auton. Syst., vol. 103, pp. 222-235, 2018.

[5] C. Yang, Y. Xie, S. Liu, and D. Sun, "Force modeling, identification, and feedback control of robot-assisted needle insertion: A survey of the literature," Sensors, vol. 18, no. 2, p. 561, 2018.

[6] I. Park, H. K. Kim, W. K. Chung, and K. Kim, "Deep learning based realtime OCT image segmentation and correction for robotic needle insertion systems," IEEE Robot. Autom. Lett., vol. 5, no. 3, pp. 4517-4524, Jul. 2020.

[7] S. Gao et al., "Force modeling of tool-tissue interaction force during suturing," in Proc. Int. Conf. Intell. Robot. Appl., 2019, pp. 297-309.

[8] K. Sorouri et al., "Utilization of a robotic mount to determine the force required to cut palatal tissue," J. Mech. Behav. Biomed. Mater., vol. 86, pp. 433-439, 2018.

[9] P. Shiet al., "A soft tissue scalpel cutting robotic system with sucker fixation," in Proc. IEEE 14th Int. Conf. Control Autom., 2018, pp. 1162-1167.

[10] F. C. Moon and T. Kalmar-Nagy, "Nonlinear models for complex dynamics in cutting materials," Philos. Trans. Roy. Soc. London. Ser. A: Math., Phys. Eng. Sci., vol. 359, no. 1781, pp. 695-711, 2001.

[11] P. Long, W. Khalil, and P. Martinet, "Modeling \& control of a meat-cutting robotic cell," in Proc. 16th Int. Conf. Adv. Robot., 2013, pp. 1-6.

[12] D. Navarro-Alarcon, Y.-H. Liu, J. G. Romero, and P. Li, "Model-free visually servoed deformation control of elastic objects by robot manipulators," IEEE Trans. Robot., vol. 29, no. 6, pp. 1457-1468, Dec. 2013. 
[13] L. Han, H. Wang, Z. Liu, W. Chen, and X. Zhang, "Vision-based cutting control of deformable objects with surface tracking," IEEE/ASME Trans. Mechatronics, vol. 26, no. 4, pp. 2016-2026, Aug. 2021.

[14] P. Long, W. Khalil, and P. Martinet, "Force/vision control for robotic cutting of soft materials," in Proc. IEEE/RSJ Int. Conf. Intell. Robots Syst., 2014, pp. 4716-4721.

[15] M. C. Gemici and A. Saxena, "Learning haptic representation for manipulating deformable food objects," in Proc. IEEE/RSJ Int. Conf. Intell. Robots Syst., 2014, pp. 638-645.

[16] Introduction to Surgical Instruments and Procedures. Gurgaon, India: Elsevier, 2008. [Online]. Available: https://books.google.ch/books?id= $8 \mathrm{cfx} 7 \mathrm{~ns} 5 \mathrm{VCIC}$

[17] M. S. Erden and A. Billard, "Hand impedance measurements during interactive manual welding with a robot," IEEE Trans. Robot., vol. 31, no. 1, pp. 168-179, Feb. 2015.

[18] E. Burdet, R. Osu, D. W. Franklin, T. E. Milner, and M. Kawato, "The central nervous system stabilizes unstable dynamics by learning optimal impedance," Nature, vol. 414, no. 6862, pp. 446-449, 2001.

[19] N. Hogan, "Impedance control: An approach to manipulation," in Proc. Amer. Control Conf., 1984, pp. 304-313.

[20] F. Ficuciello, L. Villani, and B. Siciliano, "Variable impedance control of redundant manipulators for intuitive human-robot physical interaction," IEEE Trans. Robot., vol. 31, no. 4, pp. 850-863, Aug. 2015.

[21] E. Burdet, G. Ganesh, C. Yang, and A. Albu-Schäffer, "Interaction force, impedance and trajectory adaptation: By humans, for robots," in Experimental Robotics. Berlin, Germany: Springer, 2014, pp. 331-345.

[22] N. Figueroa, S. Faraji, M. Koptev, and A. Billard, "A dynamical system approach for adaptive grasping, navigation and co-manipulation with humanoid robots," in Proc. IEEE Int. Conf. Robot. Autom., 2020, pp. 76767682 .

[23] M. Khoramshahi and A. Billard, "A dynamical system approach for detection and reaction to human guidance in physical human-robot interaction," Auton. Robots, vol. 44, pp. 1411-1429, 2020.

[24] K. Kronander and A. Billard, "Online learning of varying stiffness through physical human-robot interaction," in Proc. IEEE Int. Conf. Robot. Autom., 2012, pp. 1842-1849.

[25] A. Ajoudani, C. Fang, N. Tsagarakis, and A. Bicchi, "Reduced-complexity representation of the human arm active endpoint stiffness for supervisory control of remote manipulation," Int. J. Robot. Res., vol. 37, no. 1, pp. $155-167,2018$.

[26] A. Ajoudani, N. Tsagarakis, and A. Bicchi, "Tele-impedance: Teleoperation with impedance regulation using a body-machine interface," Int. J. Robot. Res., vol. 31, no. 13, pp. 1642-1656, 2012.

[27] J. Buchli, F. Stulp, E. Theodorou, and S. Schaal, "Learning variable impedance control," Int. J. Robot. Res., vol. 30, no. 7, pp. 820-833, 2011.

[28] W. He and Y. Dong, "Adaptive fuzzy neural network control for a constrained robot using impedance learning," IEEE Trans. Neural Netw. Learn. Syst., vol. 29, no. 4, pp. 1174-1186, Apr. 2017.

[29] W. He, C. Xue, X. Yu, Z. Li, and C. Yang, "Admittance-based controller design for physical human-robot interaction in the constrained task space," IEEE Trans. Autom. Sci. Eng., vol. 17, no. 4, pp. 1937-1949, Oct. 2020.

[30] J. E. Colgate and N. Hogan, "Robust control of dynamically interacting systems," Int. J. Control, vol. 48, no. 1, pp. 65-88, 1988.

[31] K. Kronander and A. Billard, "Passive interaction control with dynamical systems," IEEE Robot. Autom. Lett., vol. 1, no. 1, pp. 106-113, Jan. 2016.

[32] S. M. Khansari-Zadeh and A. Billard, "Learning stable nonlinear dynamical systems with Gaussian mixture models," IEEE Trans. Robot., vol. 27, no. 5, pp. 943-957, Oct. 2011.

[33] N. Figueroa and A. Billard, "Locally active globally stable dynamical systems: Theory, learning and experiments," Int. J. Robot. Res., to be published.
[34] S. M. Salehian, M. Khoramshahi, and A. Billard, "A dynamical system approach for catching softly a flying object: Theory and experiment," IEEE Trans. Robot., vol. 32, no. 2, pp. 462-471, Apr. 2016.

[35] S. S. M. Salehian and A. Billard, "A dynamical-system-based approach for controlling robotic manipulators during noncontact/contact transitions," IEEE Robot. Autom. Lett., vol. 3, no. 4, pp. 2738-2745, Oct. 2018.

[36] Z. Ghahramani and M. I. Jordan, "Supervised learning from incomplete data via an EM approach," in Proc. Adv. Neural Inf. Process. Syst., 1994, pp. $120-127$.

[37] C. E. Rasmussen, "Gaussian processes in machine learning," in Summer School on Machine Learning. Berlin, Germany: Springer, 2003, pp. 63-71.

[38] R. Wu, H. Zhang, and J. Zhao, "Robot variable impedance skill transfer and learning framework based on a simplified human arm impedance model," IEEE Access, vol. 8, pp. 225627-225638, 2020.

[39] W. Amanhoud, M. Khoramshahi, and A. Billard, "A dynamical system approach to motion and force generation in contact tasks," in Proc. Robot.: Sci. Syst., 2019.

[40] P. Mehdipour et al., "Application of Gaussian process regression (GPR) in estimating under-five mortality levels and trends in Iran 1990-2013, study protocol," Arch. Iranian Med., vol. 17, pp. 189-192, 2014.

[41] S. Baillie et al., "Comparison of a silicon skin pad and a tea towel as models for learning a simple interrupted suture," J. Veterinary Med. Educ., vol. 47, no. 4, pp. 516-522, 2020.

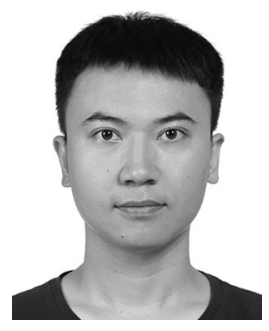

Rui Wu received the B.S. degree in mechatronics engineering from Harbin Engineering University (HEU), Harbin, China, in 2016. He is currently working toward the Ph.D. degree with the State Key Laboratory of Robotics and Systems, Harbin Institute of Technology, Harbin, China.

He was a Visiting Ph.D. student with Learning Algorithms and Systems Laboratory, École Polytechnique Fédé rale de Lausanne (EPFL), Lausanne, Switzerland, from 2019 to 2021 . His research interests include human-robot variable impedance skill transfer, learning from demonstration, robotic compliance control, and physical human-robot interaction.



Aude Billard (Fellow, IEEE) received the M.Sc. degree in physics from the Swiss Federal Institute of Technology in Lausanne (EPFL), Lausanne, Switzerland, in 1995, and the M.Sc. degree in knowledge-based systems and the Ph.D. degree in artificial intelligence from the University of Edinburgh, Edinburgh, U.K., in 1996 and 1998, respectively.

She is currently a Full Professor with the Institutes of Micro- and Mechanical Engineering and the Head of the Learning Algorithms and Systems Laboratory, School of Engineering, EPFL. Her research interests are on machine learning methods to make robot adaptive and enable learning through human guidance and practice. 